\title{
Metodología para la evaluación preliminar de la vulnerabilidad sísmica estructural de puentes de hormigón armado
}

\section{Methodology for preliminary seismic structural vulnerability assessment of concrete bridges}

\author{
$\underline{\text { D. Candebat Sánchez }}^{(*)}$, G. Godínez Melgares ${ }^{(*)}+$
}

\section{RESUMEN}

Durante eventos sísmicos fuertes muchos puentes de hormigón armado han colapsado o han sido severamente dañados causando pérdidas humanas y materiales. Se presenta una metodología para la evaluación preliminar de la vulnerabilidad sísmica estructural de puentes de hormigón armado, que garantiza la estimación adecuada de este parámetro cuando no existe suficiente información de proyecto. Se valida a partir del método de consulta de expertos Delphi, con la participación de profesionales de diversos países seleccionados por su experiencia en el tema tratado. Se obtiene una herramienta de evaluación que incluye parámetros no utilizados usualmente en las metodologías existentes y que involucra el chequeo del período de vibración de la estructura como indicador de su comportamiento global. Finalmente se presenta su aplicación en la evaluación de 18 estructuras ubicadas en la zona de mayor peligro sísmico de Cuba.

Palabras claves: Metodología, vulnerabilidad sísmica estructural, puentes, hormigón.

\section{ABSTRACT}

During strong seismic events many highway reinforced concrete bridges have collapsed or have been severely damaged causing human and material losses. A methodology for preliminary assessment of seismic structural vulnerability of reinforced concrete bridges which ensures adequate estimation of this parameter without enough project information is presented. It is validated using the Delphi method, with the participation of professionals from different countries, which were selected for their experience in this topic. An evaluation tool is obtained including parameters not usually used by the existing methodologies and involving the checking of vibration period of the structure as an indicator of its overall behavior. Finally, the assessment of 18 structures located in the zone of higher seismic hazard of Cuba is presented.

Keywords: Methodology, seismic structural vulnerability, bridges, concrete.

(*) Centro Nacional de Investigaciones Sismológicas. Santiago de Cuba, (Cuba)

Persona de contacto/Corresponding author: dario@cenais.cu (D. Candebat Sánchez) ORCID: http://orcid.org/oooo-0002-0066-4937 (D. Candebat Sánchez)

Cómo citar este artículo/Citation: Candebat Sánchez, D., Godínez Melgares, G. (2018). Metodología para la evaluación preliminar de la vulnerabilidad sísmica estructural de puentes de hormigón armado. Informes de la Construcción, 70(549): e247. https://doi. org/10.3989/id.55418.

Copyright: (C) 2018 CSIC. Este es un artículo de acceso abierto distribuido bajo los términos de la licencia de uso y distribución Creative Commons Reconocimiento 4.0 Internacional (CC BY 4.0). 


\section{INTRODUCCIÓN}

En sismos fuertes, como los ocurridos en haití el 12 de enero y en chile el 27 de febrero, ambos en el año 2010, se puso de manifiesto la elevada vulnerabilidad que presentan los puentes ante las acciones generadas por sismos fuertes. Varias estructuras colapsaron afectando las acciones necesarias a acometer posteriormente a estos eventos.

Los daños mostrados por estas obras de fábrica han marcado la pauta para el desarrollo de nuevos estudios. (1), (2), (3), (4) y (5), entre otros autores, han señalado las principales deficiencias que han propiciado la ocurrencia de daños y hasta el colapso de puentes en varios países del mundo, demostrando la necesidad de estudiar detalladamente su comportamiento, pues sólo la prevención y el empeño en la rehabilitación garantizan un desempeño adecuado.

Como parte de los estudios dirigidos a la mitigación de desastres, internacionalmente han surgido numerosas metodologías y procedimientos encaminados a verificar el comportamiento de los puentes ante terremotos moderados y fuertes. En cuba, específicamente en la región suroriental, se han realizado varios trabajos de evaluación de la vulnerabilidad sísmica estructural de puentes de carreteras. Con este propósito (6) utiliza una metodología la cual, a partir de un análisis modal espectral de dos puentes prefabricados emplazados en carreteras de interés nacional, le permite demostrar la elevada vulnerabilidad que estos poseen. Desarrollado en japón, el procedimiento elaborado por (1) fue empleado en la evaluación ante multiamenazas de las carreteras de interés nacional de santiago de cuba, investigación que demuestra, que aunque en cuba no se han tenido reportes de daños en puentes debido a sismos de gran intensidad, es imprescindible realizar estudios dirigidos a determinar los problemas que inciden en el mal comportamiento de estos, sobre todo al considerar el escenario de la zona suroriental de cuba, clasificada como de mayor peligrosidad sísmica del país.

Estos estudios han puesto de manifiesto además, la necesidad de contar con una metodología propia que permita obtener resultados preliminares acerca del comportamiento de los puentes, debido a que la utilización de procedimientos extranjeros supone la adopción de criterios para adaptarlos a las condiciones cubanas que introducen incertidumbres en los resultados obtenidos. Igualmente, las metodologías estudiadas y utilizadas para el análisis de la vulnerabilidad de los puentes en cuba, no consideran algunos parámetros que, en opinión de los autores, son indicadores del probable comportamiento de la estructura ante acciones sísmicas.

Estos elementos fundamentan la propuesta de una metodología que incluya aquellos parámetros que han demostrado ser la causa de daños importantes en puentes ubicados en países afectados por sismos fuertes, por lo que en este trabajo se presenta una herramienta para la evaluación preliminar de la vulnerabilidad sísmica estructural de puentes de hormigón armado, que puede ser utilizada en cualquier zona sísmica del mundo. La primera versión validada por expertos fue empleada por (7), en el año 2012, para la evaluación del puente sobre el río arroyito ubicado en la autopista nacional de santiago de cuba. Se presenta una versión actualizada a partir del criterio de nuevos expertos que propicia la inclusión de parámetros novedosos en el análisis. Asimismo se introduce el criterio de parámetros invalidantes, elemento que propor- ciona mayor relevancia a los aspectos que por sí solos constituyen indicativos de una elevada vulnerabilidad estructural. Esta metodología, además de garantizar una estimación preliminar de la vulnerabilidad sísmica, permite establecer niveles de prioridad en el proceso de rehabilitación de un conjunto de estructuras. Para seleccionar el método de investigación utilizado se tuvieron en cuenta los criterios aportados por (8) en su análisis de la vulnerabilidad sísmica de puentes.

\section{MATERIALES Y MÉTODOS}

Para proponer los parámetros a evaluar como parte de esta metodología se divide el análisis en dos etapas:

1. Estudio de las causas de daños en puentes de hormigón en países afectados por sismos fuertes.

2. Análisis de metodologías existentes a nivel internacional para la evaluación de la vulnerabilidad sísmica estructural de puentes.

Como resultado del análisis de las principales causas de daños se constata que (3) y (9), al evaluar las afectaciones en puentes provocadas por sismos fuertes ocurridos en el mundo, identifican las causas más comunes del colapso de la mayoría de estas estructuras. (9) manifiesta que en eventos recientes, los daños típicos pueden ser asociados a cuatro grupos fundamentales:

- Pérdida de apoyo de los tramos debido a longitudes de apoyo insuficientes.

- Colapso y daños en superestructuras debido a tableros inadecuados, llaves de cortante deficientes y desbalance de la rigidez de las pilas.

- Fallo de columnas por flexión y cortante debido a un inadecuado detallado y baja ductilidad.

- Fallo estructural debido a fallo del terreno, licuación y ruptura de fallas.

Según estos autores, las lecciones aprendidas de estos terremotos han propiciado los principales avances en la ingeniería sísmica de puentes.

Por su parte, la investigación realizada por (3) también menciona las afectaciones descritas por (9) entre los daños más comúnmente observados. Sin embargo incluyen además el golpeteo de estructuras del puente debido a la previsión de bajos valores de desplazamientos sísmicos que conduce a la consideración de dimensiones de juntas inadecuadas entre elementos adyacentes; las fallas en cabezales a partir de considerar poca capacidad al cortante, terminación prematura del refuerzo negativo del cabezal e insuficiente anclaje del refuerzo del cabezal en las regiones finales; las fallas en nudos debido a que el refuerzo utilizado ha sido inadecuado y las fallas en los cimientos como resultado de la poca resistencia a flexión debido a la omisión del refuerzo superior, poca resistencia al cortante en la región inmediatamente debajo de la pila, la cual está sometida a grandes esfuerzos de este tipo, insuficiente anclaje, escaso desarrollo del refuerzo de la pila e incorrecta conexión entre las pilas y el cimiento.

En su investigación, (2) demuestran que muchos de los puentes dañados en japón durante el terremoto de hyogo - ken - nanbu (kobe) en 1995, incluyendo algunos que llegaron al colapso total, habían sido diseñados con criterios de acuerdo con las especificaciones japonesas de diseño de puentes 
de carreteras de 1964 y 1971. Estos autores explican que las acciones sísmicas derivadas de este evento fueron significativamente mayores a cualquiera de las consideradas en el diseño, al igual que las acciones verticales que fueron extremadamente grandes.

Asimismo (5) al realizar un análisis de las afectaciones ocasionadas a los puentes chilenos, debido al terremoto del $27 \mathrm{de}$ febrero del 2010, expresa que las causas de estos daños están relacionadas con:

- Ausencia de vigas transversales (diafragmas) para soportar adecuadamente la fuerza sísmica transversal al puente e impedir la caída de los tramos.

- El diseño inadecuado de los topes transversales.

- La presencia del fenómeno de socavación.

- Deficiente construcción del anclaje vertical.

En relación con la revisión de metodologías existentes, se identificaron algunas que utilizan índices para la evaluación y, por tanto, incorporan parámetros de evaluación primaria. Se analizaron las metodologías siguientes:

- Método de inspección de puentes de carreteras (1).

- Modelo de evaluación del índice de vulnerabilidad sísmica basada en conjuntos difusos (8).

- Metodología desarrollada por la agencia federal de manejo de emergencia (fema) (10).

- Metodología para la evaluación de la vulnerabilidad sísmica de puentes carreteros (11).

En general estos procedimientos tratan aspectos que inciden de manera directa en el desempeño de los puentes de uno u otro material y los más exactos tratan de considerar el comportamiento no lineal de la estructura.

Asimismo, (12) realizan una revisión de métodos existentes para la evaluación de la vulnerabilidad sísmica de puentes e identifican qué parámetros son usualmente empleados por sus autores (tabla 1).

Relacionando los parámetros identificados y mostrados por (12) en la tabla 1, con las afectaciones que comúnmente han manifestado estas estructuras durante la ocurrencia de sismos fuertes, se puede comprobar que estos están intrínse- camente relacionados con los aspectos que han constituido causa de daños. Por lo tanto, su análisis previo permite establecer con qué nivel de probabilidad las estructuras evaluadas pueden ser afectadas por eventos de este tipo. Igualmente los parámetros considerados por (1), (8), (10) y (11) están incluidos en los identificados por (12).

Como resultado de este análisis se definen los parámetros que son usualmente utilizados por los especialistas para evaluar la vulnerabilidad sísmica de puentes. Sin embargo, teniendo en cuenta los daños observados, existen algunos elementos que no son tomados en cuenta por los métodos estudiados y que intervienen en el comportamiento de estas estructuras, por lo que son incluidos en la metodología propuesta, a decir:

- La evaluación de la calidad de los materiales de construcción de la estructura.

- La existencia de trabas o topes sísmicos y diafragmas que garanticen la estabilidad de los tramos.

- La rigidez global de la estructura expresada a través de su valor de período natural de vibración.

- La probabilidad de ocurrencia del fenómeno de resonancia entre la estructura y el suelo. Según (13), un estudio realizado por gozar en 2007 concluyó que los grandes daños producidos por los terremotos de 1998 y 2004 en la región de bovec, eslovenia, fueron consecuencia de la amplificación local del sitio y de la condición de resonancia sueloestructura.

- La falta de homogeneidad del suelo, que propicia variación en su respuesta ante las acciones sísmicas y, por tanto, modifica el comportamiento de la estructura, principalmente en puentes continuos, de longitud significativa y con detalles estructurales especiales. Según (14), la variación espacial del movimiento del suelo entre pilas del puente puede afectar significativamente su respuesta.

- La precisión de la cercanía de fallas geológicas: su proximidad presupone alta probabilidad de daños en las estructuras construidas en la zona. (14) establece que cuando la estructura está ubicada a una distancia menor de 6 millas $(9.656 \mathrm{~km})$ de una falla activa, debe realizarse un análisis específico del sitio.

- El análisis de la relación de la esbeltez de las columnas de pilas y estribos y no de la altura de estas, teniendo en cuenta que la altura, como dimensión independiente, no refleja cuán susceptibles de daños son estos elementos.

Tabla 1. Parámetros que se emplean en distintas metodologías para estimar la vulnerabilidad sísmica de puentes.

\begin{tabular}{|l|l|}
\hline \multicolumn{2}{|c|}{ Parámetros } \\
\hline Longitud de apoyos en pilas y estribos & Tipo de cimentación \\
\hline Tipo de superestructura & Aceleración máxima del terreno \\
\hline Año de construcción (normas usadas) & Tipo de estribos \\
\hline Ángulo de esviaje & Longitud del puente \\
\hline Material de las pilas & Ancho del puente \\
\hline Refuerzo en pilas & Promedio de tráfico diario \\
\hline Efectos de sitio & Altura de estribos \\
\hline Tipos de apoyo & Refuerzo de la cimentación \\
\hline Potencial de licuefacción & Longitud efectiva de columnas \\
\hline Irregularidad en rigidez & Dimensión transversal de columnas \\
\hline Rehabilitaciones previas & Asentamiento de terraplenes de acceso \\
\hline Número de claros & Estado de conservación del puente \\
\hline Tipo de subestructura & Procedimiento constructivo \\
\hline No. De articulaciones internas & Elementos no estructurales \\
\hline
\end{tabular}


La inclusión de estos parámetros permite identificar aquellos aspectos que afectan el comportamiento estructural global y facilitan la posterior evaluación detallada de las estructuras con un mayor nivel de vulnerabilidad preliminar.

Sobre la base de este análisis se elabora una metodología que contiene 23 aspectos a considerar en la evaluación, divididos en tres grupos:

\section{Superestructura.}

2. Subestructura.

3. Condiciones del sitio.

Cada uno de los parámetros fue ordenado y cuantificado según su incidencia en el comportamiento de la estructura, posteriormente, cada grupo (superestructura, subestructura y condiciones del sitio) fue evaluado según su incidencia en su desempeño global. Esta asociación de los parámetros a cada grupo permite personalizar el análisis y otorgar un peso superior a aquellos aspectos que poseen mayor incidencia en cada uno.

Para validar el procedimiento propuesto se aplicó el método de consulta de expertos delphi que, según (15), es un «método de estructuración de un proceso de comunicación grupal que es efectivo a la hora de permitir a un grupo de individuos, como un todo, tratar un problema complejo» (p. 3). Por su parte, (16) afirma que «el método delphi permite estructurar un proceso comunicativo de diversos expertos organizados en grupo-panel con vistas a aportar luz en torno a un problema de investigación» (p. 4). En esencia, consiste en una consulta a un panel de expertos de forma individual a través de la aplicación sucesiva de cuestionarios para generar convergencias de opiniones.

Consta de cuatro fases fundamentales:

- Definición de objetivos: donde se define el problema y el objetivo general que se quiere lograr con la aplicación del método.

- Selección del panel de expertos teniendo en cuenta el objetivo definido, la experiencia, acceso a la información y disponibilidad. Al decir de (17), este es uno de los elementos que condicionan el éxito del método pues, siendo el grupo de expertos la fuente de información, se entiende la importancia que tiene su correcta selección y participación.

- Elaboración y entrega de los cuestionarios que faciliten la respuesta de los encuestados.
- Análisis de resultados, realización de las rondas necesarias para disminuir la dispersión y precisar la opinión media consensuada.

Para la confección de las encuestas se creó un grupo de trabajo formado por 5 especialistas e investigadores del centro nacional de investigaciones sismológicas y la universidad de oriente de santiago de cuba. Se garantizó la elaboración de un documento para facilitar las respuestas de los expertos a consultar y que incluyó los parámetros y la evaluación propuesta. Los cuestionarios fueron enviados vía e-mail y las valoraciones realizadas por el panel fueron recibidas por la misma vía.

Posteriormente se determina la cantidad de especialistas a consultar según las respuestas positivas recibidas. El análisis arroja la necesidad de conformar un panel de 14 expertos, a los cuales les son enviadas las encuestas, que posteriormente fueron procesadas por el grupo de trabajo anteriormente mencionado. Los expertos seleccionados pertenecen a centros de investigación, universidades y entidades de diseño cubanos y extranjeros (tabla 2).

Se realizaron tres rondas garantizando finalmente la coincidencia de más del $75 \%$ de los expertos, lo que aseguró que el resultado final incluyera la mayoría de sus opiniones.

\section{RESULTADOS Y DISCUSIÓN}

El análisis realizado y la validación con los expertos, permitieron obtener un procedimiento para evaluar preliminarmente la vulnerabilidad sísmica estructural de puentes de hormigón armado construidos en cualquier región con sismicidad moderada o alta sin contar con información de proyecto, aspecto que en muchas ocasiones impide el análisis.

Se incluyeron parámetros que inciden de manera significativa en el comportamiento de estas estructuras, de forma que, aún sin poder aplicar un procedimiento analítico (detallado), sea posible obtener resultados cercanos al probable desempeño estructural.

La novedad de la metodología propuesta radica en la inclusión de varios parámetros que no han sido considerados por las metodologías analizadas, dígase la degradación de la rigidez, la existencia de trabas sísmicas y diafragmas, la esbeltez de las columnas de pilas y estribos, la posibilidad de resonancia suelo-estructura, la homogeneidad del suelo y la cercanía de

Tabla 2. Distribución por institución y país de los expertos integrantes del panel

\begin{tabular}{|l|l|l|}
\hline \multicolumn{1}{|c|}{ Institución } & \multicolumn{1}{c|}{ País } \\
\hline Universidad de oriente & Cuba \\
\hline Empresa de proyecto \# 15 & Cuba \\
\hline Empresa de proyecto vértice & Cuba \\
\hline Empresa de proyecto \# 2 (empai) & Cuba \\
\hline Universidad autónoma metropolitana & México \\
\hline $\begin{array}{l}\text { Oficina nacional de evaluación sísmica, vulnerabilidad de infraestructura } \\
\text { y edificaciones }\end{array}$ & República Dominicana \\
\hline Universidad autónoma de méxico & México \\
\hline Centro nacional de investigaciones sismológicas & Cuba \\
\hline Universidad de berkeley. Eua & Eua \\
\hline Total & \multicolumn{1}{|c|}{$\mathbf{2}$} \\
\hline
\end{tabular}


fallas geológicas; asimismo también se sustenta en la utilización del período fundamental de vibración como indicador del comportamiento global de la estructura y en el análisis de su relación con la rigidez del suelo del área de emplazamiento.

Igualmente, un aspecto novedoso lo constituye la definición de los parámetros invalidantes, que permiten definir elevados niveles de vulnerabilidad sísmica estructural a partir del análisis de determinados elementos que de manifestarse, provocan un inadecuado comportamiento de los puentes ante acciones generadas por eventos sísmicos moderados y fuertes. En la tabla 3 se muestra la distribución realizada.

\section{Dónde:}

Tp - período de la estructura, determinado con mediciones instrumentales (segundos).

Tc - período de vibración fundamental de la estructura calculado con ecuaciones empíricas (segundos).

Lg - esbeltez geométrica de las columnas del puente. Calculada según (13).
$\operatorname{Lg}=\operatorname{lp} / \mathrm{hp}$.

Hp - profundidad de la sección (m)

$\ell p$ - longitud de pandeo (m). $\ell p=a \ell$.

$\ell$ - altura libre de la columna (m).

A - factor de longitud de pandeo. Se calcula según (18).

Cada uno de los parámetros fue ordenado y cuantificado según su incidencia en el comportamiento de la estructura (tabla 4), posteriormente, cada grupo fue evaluado según su importancia en el desempeño global, como sigue:

Ivse $=(25 \%($ vspe $)+45 \%($ vsbe $)+30 \%($ vst $)) / 100 \%$

Dónde:

Ivse - índice de vulnerabilidad sísmica estructural.

Vspe - vulnerabilidad sísmica estructural de la superestructura del puente.

Tabla 3. Parámetros evaluados.

\begin{tabular}{|c|c|c|c|}
\hline Aspectos a evaluar referidos a la superestructura & Evaluación & Aspectos a evaluar referidos a la subestructura & Evaluación \\
\hline Especificaciones de diseño & 2.00 & Degradación de la rigidez & 2.50 \\
\hline Después de 1999 & 1.00 & $\mathrm{Tp} \leq \mathrm{tc}$ & 0.00 \\
\hline Antes de 1999 & 2.00 & $\mathrm{Tp} \geq \mathrm{tc}$ & 2.50 \\
\hline Tipo de superestructura & 1.50 & Estado técnico de la subestructura & 2.00 \\
\hline Isostática con losa continua & 0.75 & Muy buena y buena (sin daños en pilas y/o estribos) & 0.00 \\
\hline Isostática & 1.50 & Regular (daños moderados en pilas y/o estribos) & 1.00 \\
\hline Longitud de apoyo & 1.50 & Mala y muy mala (daños extensivos en pilas y/o estribos) & 2.00 \\
\hline Cumple lo especificado en la norma vigente & 0.00 & Tipo de subestructura & 2.00 \\
\hline No cumple & 1.50 & pilas & \\
\hline Existencia de trabas sísmicas & 1.00 & muros de corte & 0.50 \\
\hline Existen y poseen diseño satisfactorio & 0.00 & pórticos & 0.75 \\
\hline Existen pero presentan diseño inadecuado & 0.75 & mono columnas o pilas en v o inclinadas & 1.00 \\
\hline No existen & 1.00 & estribos & \\
\hline Existencia de diafragmas (vigas transversales) & 1.00 & abiertos & 0.50 \\
\hline Posee diafragmas & 0.00 & cerrados con drenaje adecuado & 0.75 \\
\hline No posee diafragmas & 1.00 & cerrados sin drenaje adecuado & 1.00 \\
\hline Dispositivos de apoyo & 1.00 & Esbeltez geométrica de columnas de pilas y estribos (adimensional) & 1.00 \\
\hline Existen & 0.00 & $\lg \leq 6.5$ & 0.00 \\
\hline Existen en mal estado y/o son inadecuados & 0.75 & $6.5 \leq \lg \leq 30$ & 0.75 \\
\hline No existen & 1.00 & $\lg >30$ & 1.00 \\
\hline Trazado de la superestructura & 1.00 & Esviaje & 1.00 \\
\hline Recto & 0.00 & Sin esviaje o con ángulo menor de $15^{\circ}$ & 0.00 \\
\hline Curva horizontal menor de $90^{\circ}$ y esviaje menor de $30^{\circ}$ & 0.50 & Esviaje entre $15^{\circ}$ y $30^{\circ}$ & 0.50 \\
\hline Curva de $90^{\circ}$ y esviaje entre $30^{\circ}$ y $45^{\circ}$ & 0.75 & Esviaje entre $31^{\circ}$ y $45^{\circ}$ & 0.75 \\
\hline Curva mayor de $90^{\circ}$ y esviaje mayor de $45^{\circ}$ & 1.00 & Esviaje mayor de $45^{\circ}$ & 1.00 \\
\hline Curvas verticales & 0.50 & Unión columna - cimentación & 1.00 \\
\hline No tiene & 0.00 & Unión monolítica & 0.00 \\
\hline$<6 \%$ & 0.25 & No existe monolistismo & 1.00 \\
\hline$>6 \%$ & 0.50 & Probabilidad de resonancia estructura - suelo & 0.50 \\
\hline Posibilidades de golpeteo & 0.50 & Tsuelo $\neq$ t estructura & 0.00 \\
\hline Separación adecuada entre estructuras adyacentes & 0.00 & Tsuelo $=$ t estructura & 0.50 \\
\hline Separación menor a la requerida a la misma altura & 0.25 & & \\
\hline Separación menor a la requerida a diferentes alturas & 0.50 & & \\
\hline Evaluación total & 10.00 & Evaluación total & 10.00 \\
\hline
\end{tabular}


Tabla 4. Ponderación de parámetros según su incidencia en la estructura.

\begin{tabular}{|c|c|}
\hline $\begin{array}{c}\begin{array}{c}\text { Aspectos a evaluar referidos a las condiciones } \\
\text { del sitio }\end{array} \\
\text {. }\end{array}$ & Evaluación \\
\hline Aceleración del sitio (\% gravedad) & 2.50 \\
\hline $0,075 \leq a<0,10$ & 0.00 \\
\hline $0,10 \leq \mathrm{a}<0,20$ & 0.50 \\
\hline $0,20 \leq a<0,30$ & 1.75 \\
\hline $\mathrm{A} \geq 0,30$ & 2.50 \\
\hline Erosión y/o socavación & 2.00 \\
\hline Poco probable & 0.00 \\
\hline Probable & 1.00 \\
\hline Existe en estado incipiente & 1.50 \\
\hline Existe y afecta la estabilidad del puente & 2.00 \\
\hline Riesgos geológicos (licuefacción) & 2.00 \\
\hline Insignificante & 0.00 \\
\hline Media & 0.75 \\
\hline Alta & 1.50 \\
\hline Muy alta & 2.00 \\
\hline Dureza de los suelos & 1.50 \\
\hline Duros (s1) & 0.00 \\
\hline Menos duros (s2) & 0.50 \\
\hline Blandos (s3) & 0.75 \\
\hline Muy blandos(s4) & 1.50 \\
\hline Irregularidad topográfica del suelo & 1.00 \\
\hline No & 0.00 \\
\hline $\mathrm{Si}$ & 1.00 \\
\hline Homogeneidad del suelo & 0.50 \\
\hline Sin variación entre eje de pilas y estribos & 0.00 \\
\hline Variable & 0.50 \\
\hline Existencia de fallas geológicas & 0.50 \\
\hline No existen en la zona o están ubicadas a más de $50 \mathrm{~km}$ & 0.00 \\
\hline Existen fallas pasivas ubicadas a menos de $50 \mathrm{~km}$ & 0.25 \\
\hline Existen fallas activas ubicadas a menos de $50 \mathrm{~km}$ & 0.50 \\
\hline Evaluación total & 10.00 \\
\hline
\end{tabular}

Vsbe - vulnerabilidad sísmica estructural de la subestructura del puente.

Vst - vulnerabilidad asociada al sitio de emplazamiento.

En la tabla 5 se muestran los intervalos para la evaluación de la vulnerabilidad.

El intervalo de evaluación final se obtuvo evaluando de forma aleatoria todos los factores con los valores medios obteniendo el rango para la vulnerabilidad moderada.

Otro elemento significativo de esta metodología lo constituye la consideración de variables que por sí solas o combinadas con otras determinan una alta vulnerabilidad de la estructura. Estas variables, denominadas invalidantes, se relacionan a continuación:

La combinación del no cumplimiento de la longitud de apoyo con la ausencia de trabas sísmicas o diafragmas o ambos. Esto se debe a que aún cuando algunos tramos se apoyan en los cabezales una longitud menor que la establecida por los códigos de diseño vigentes la existencia de las trabas o topes sísmicos y diafragmas puede impedir la caída de los tramos.

- Degradación de la rigidez.

- Estado técnico de la subestructura malo y muy malo.
Tabla 5. Rangos para la evaluación.

\begin{tabular}{|l|l|}
\hline Evaluación & \multicolumn{1}{|c|}{ Vulnerabilidad } \\
\hline $0.00-0.25$ & Baja \\
\hline $0.26-0.45$ & Moderada \\
\hline $0.46-1.00$ & Alta \\
\hline
\end{tabular}

- Existencia de socavación que afecta la estabilidad de la estructura.

- Resonancia suelo - estructura

Esta metodología ha sido utilizada para el análisis de la vulnerabilidad sísmica estructural de los puentes ubicados en las carreteras de mayor envergadura de santiago de cuba: carretera granma y autopista nacional, obteniendo resultados que han permitido prevenir a los administradores viales sobre la posible ocurrencia de daños, resultados que han sido validados además, en la medida de lo posible, por análisis más detallados, utilizando software de análisis estructural, en los casos en que se cuenta con proyecto técnico ejecutivo. Se muestra su aplicación a 18 puentes prefabricados de hormigón armado y pretensado, construidos en la región de mayor peligrosidad sísmica de cuba.

Los puentes estudiados han sido erigidos con la tipología prefabricada cubano-italiana, entre los años 1973 y 1988. A continuación se describen algunas de las características de estas estructuras que favorecen elevados niveles de vulnerabilidad sísmica estructural.

La utilización de un apoyo móvil y uno fijo en cada luz de la estructura caracteriza la tipología analizada, la cual genera puentes isostáticos en la dirección longitudinal con poca redundancia; por tanto, con inadecuada transmisión de la carga sísmica entre los tramos de la superestructura (figura 1).

En el caso de las pilas y estribos prefabricados, sus cabezales están conformados por piezas prefabricadas de hormigón armado. Entre estos elementos se crean uniones articuladas debido a la ausencia de un refuerzo longitudinal que garantice la continuidad y, por tanto, la transmisión de las solicitaciones (figura 2).

De igual forma, en la figura 3 se observa que la unión cabezal prefabricado-columna prefabricada es articulada y se realiza con cuatro barras de acero, por lo que esta unión constituye el punto menos resistente a esfuerzo cortante del sistema.

En las figuras 4-10 se muestran imágenes que reflejan algunos problemas detectados en inspecciones técnicas realizadas a los puentes estudiados.

En la tabla 6 se relaciona el estado técnico de cada estructura, determinado según los criterios de (20). En la tabla 7 se muestra la información estructural básica utilizada en el análisis.

En estos resultados influyen en gran medida las condiciones del tablero de las estructuras, observándose juntas en mal estado, daños en la carpeta asfáltica, roturas en los pretiles, fallos de losas de hormigón armado y aparatos de apoyo dañados. Igualmente inciden la presencia de socavación incipiente al pie de pilas y la erosión del material del aproche. 

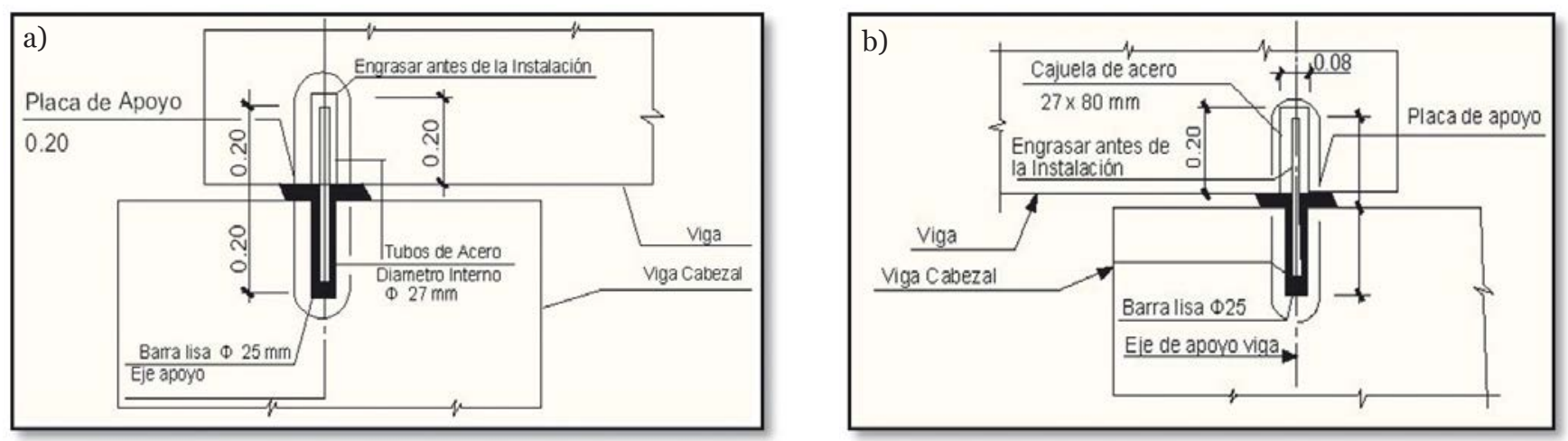

Figura 1 a y b. Detalle de unión Viga-Cabezal. a) Sección de apoyo fijo. b) Sección de apoyo móvil. Fuente: (19)

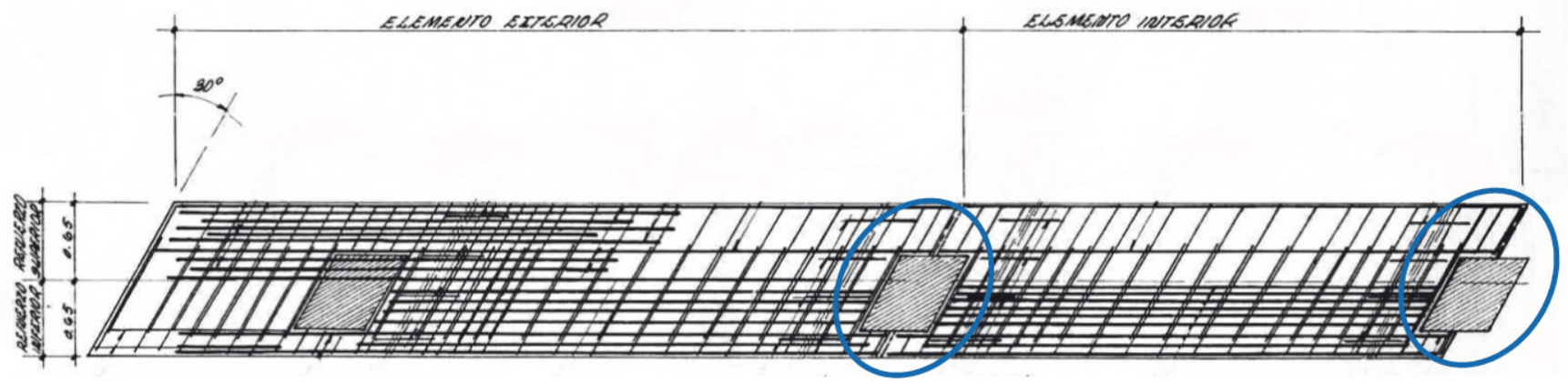

Figura 2. Uniones articuladas entre piezas prefabricadas que conforman el cabezal de pilas y estribos. Fuente: (19).

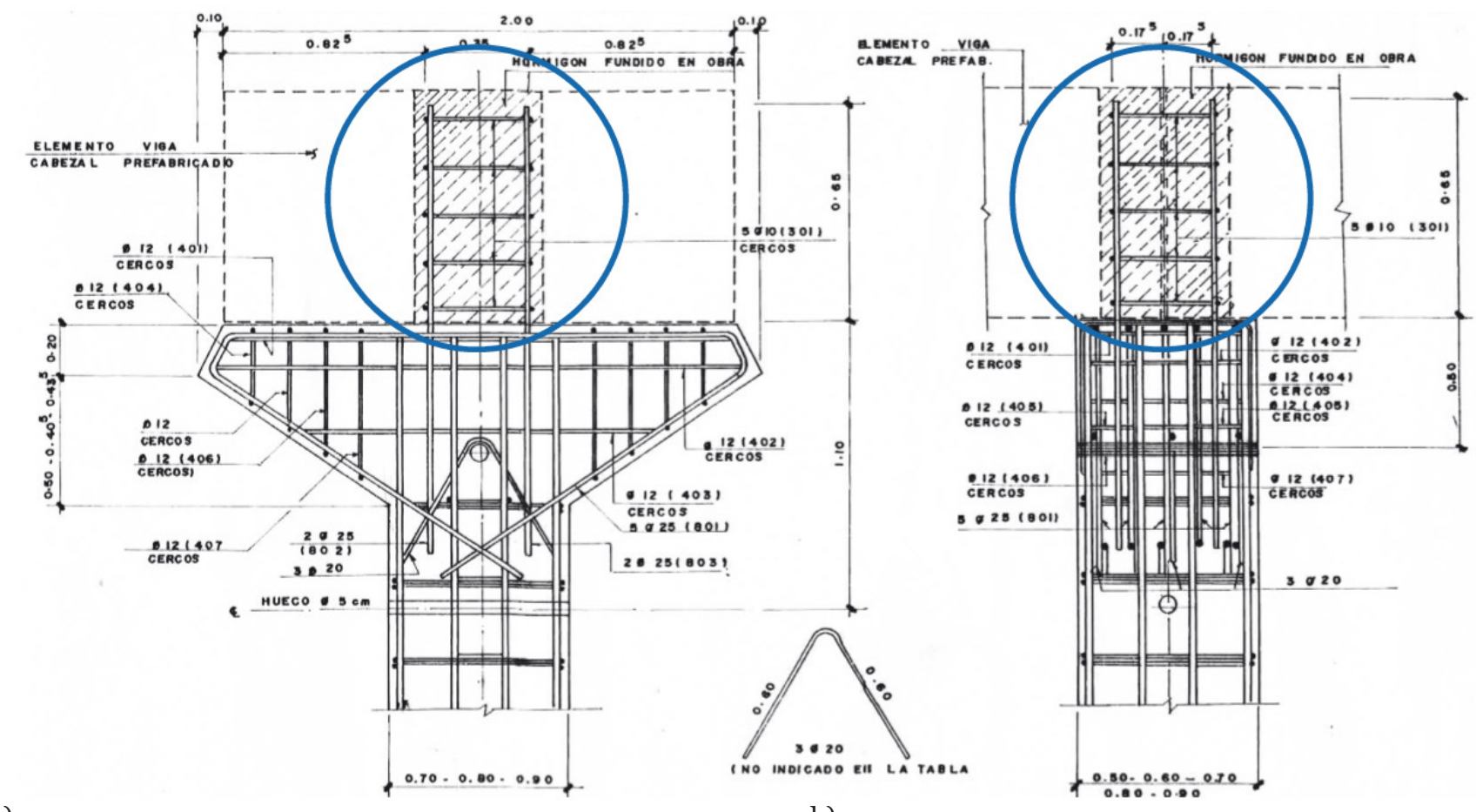

a)

b)

Figura 3. Unión columna prefabricada - cabezal prefabricada. a) Vista frontal. b) Vista lateral. Fuente: (19).

Como se muestra en la tabla 8 los puentes estudiados manifiestan vulnerabilidad entre Moderada y Alta, constatándose que el $56 \%$ posee una alta vulnerabilidad.

A partir del análisis de los resultados de cada grupo de parámetros evaluados (superestructura, subestructura y condiciones del sitio) se constata que:
En contraposición con otras metodologías, la división en tres grupos permite definir cuales parámetros están influyendo en mayor medida en el resultado final. En este caso se observa como los puentes manifiestan, en general, una elevada vulnerabilidad sísmica asociada a las características de su superestructura, sin embargo, el hecho de poseer subestructuras tipo pórticos, con buen estado técnico y con condiciones de rigi- 


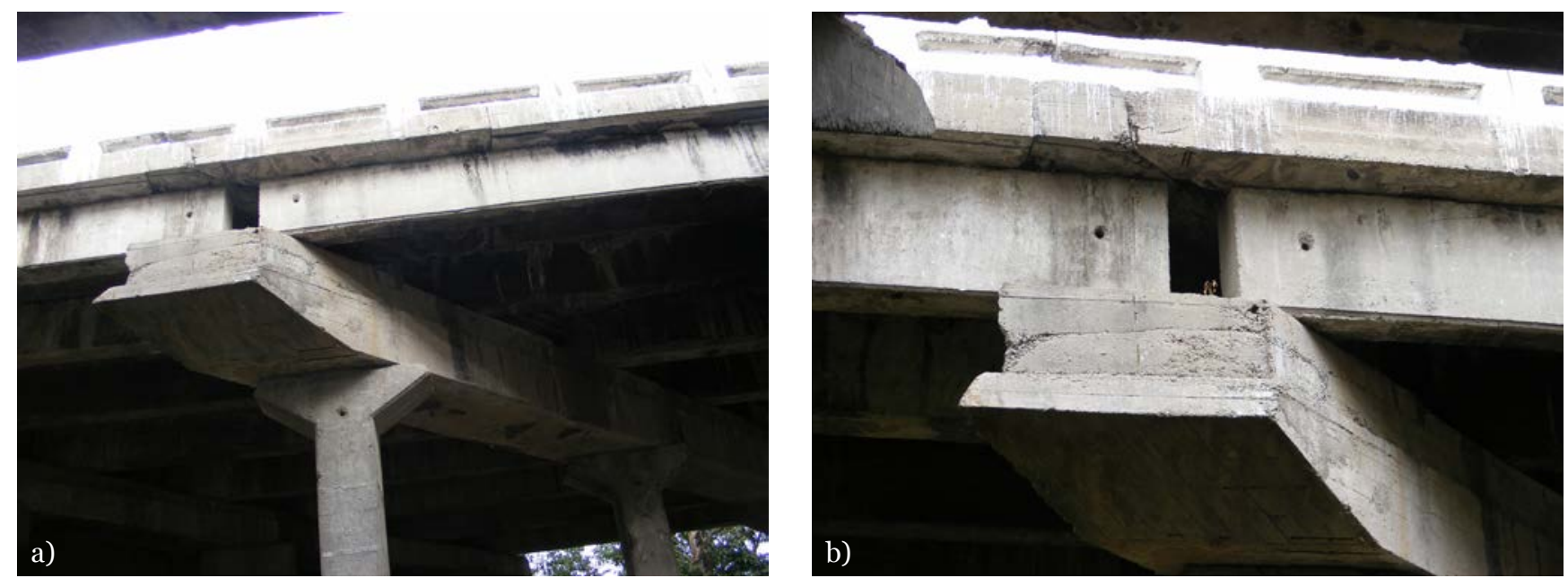

Figura 4 a y b. Longitudes de apoyo inadecuadas sobre cabezales de pilas.
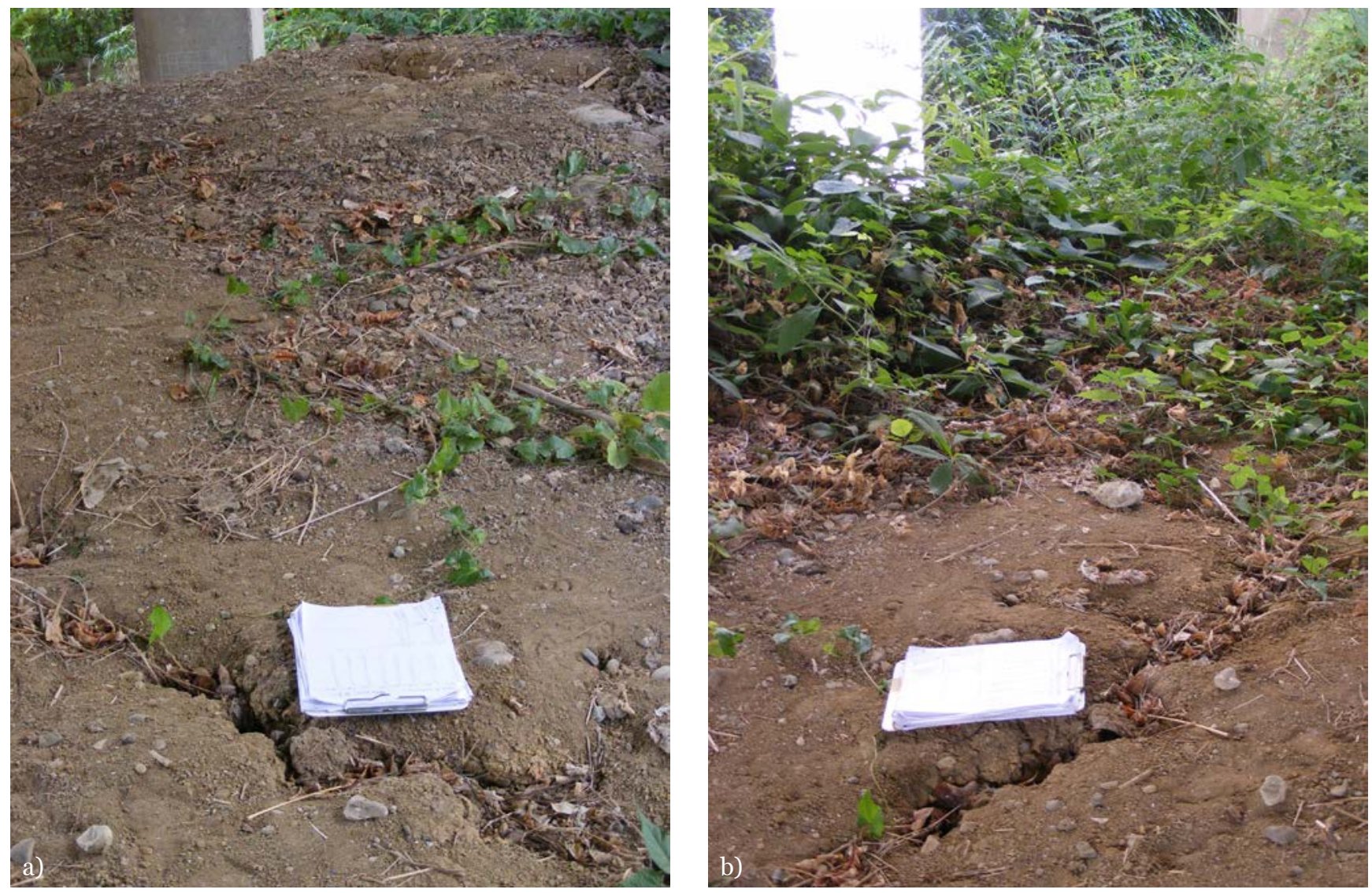

Figura 5 a y b. Grieta en corona del talud, evidenciando la existencia de pequeños deslizamientos.

dez adecuadas, propicia una vulnerabilidad asociada a este elemento entre baja y moderada, por lo que la vulnerabilidad final se ve modificada. Esto constituye otra de las novedades de la herramienta de evaluación propuesta.

Este análisis permite a proyectistas e investigadores centrar la atención en aquellos elementos que deben ser priorizados para disminuir el nivel de vulnerabilidad de la estructura.

Los factores que fundamentalmente incidieron en los elevados niveles de vulnerabilidad detectados son:
- Fecha de diseño y construcción: aspecto que refleja la ausencia de criterios actualizados en relación al diseño sismorresistente (todas las estructuras fueron diseñadas y construidas antes de 1999, fecha de aprobación del código de diseño sismorresistente cubano vigente).

- Superestructuras isostáticas (simplemente apoyadas): esta característica define la existencia de estructuras poco redundantes.

- Incumplimiento de la longitud de apoyo, sin trabas sísmicas ni diafragmas: este parámetro es invalidante, lo que significa que las estructuras que no poseen longitudes de apoyo según lo establecido por (21) automáticamente son 


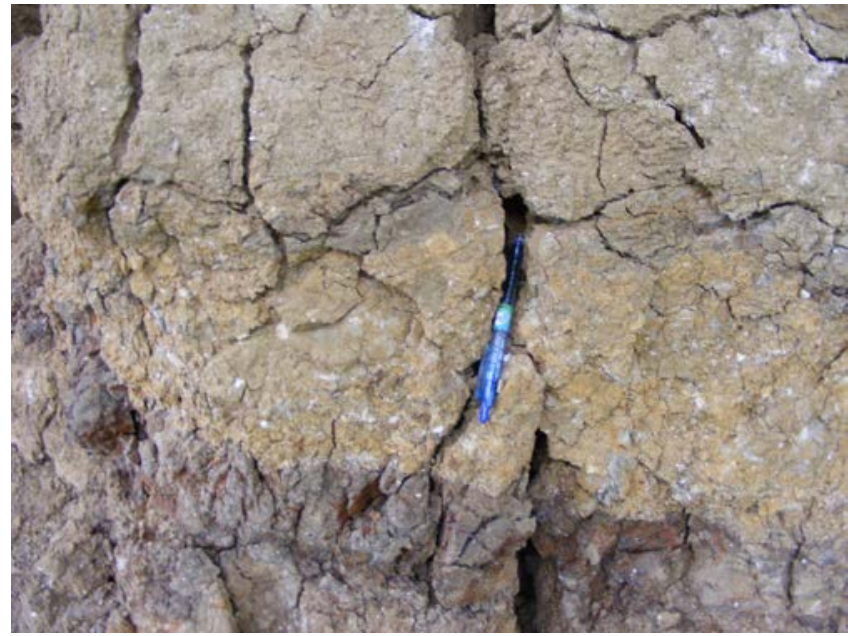

Figura 6. Grietas por desecación en el talud del cauce del río, formado por suelos arcillosos expansivos. Desprendimiento del material arcilloso. Destrucción parcial del talud.

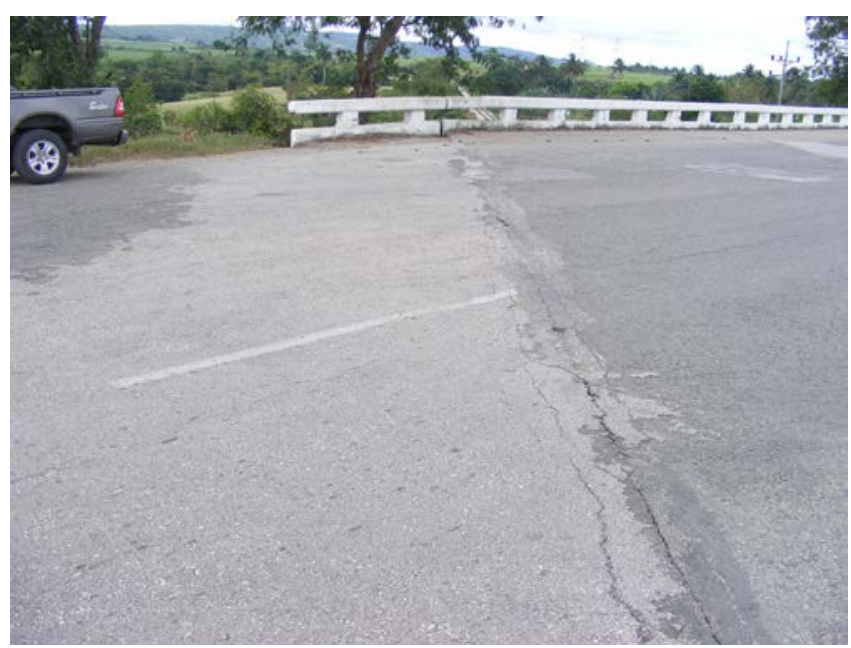

Figura 8. Hundimiento de la losa de aproche en ambos extremos.

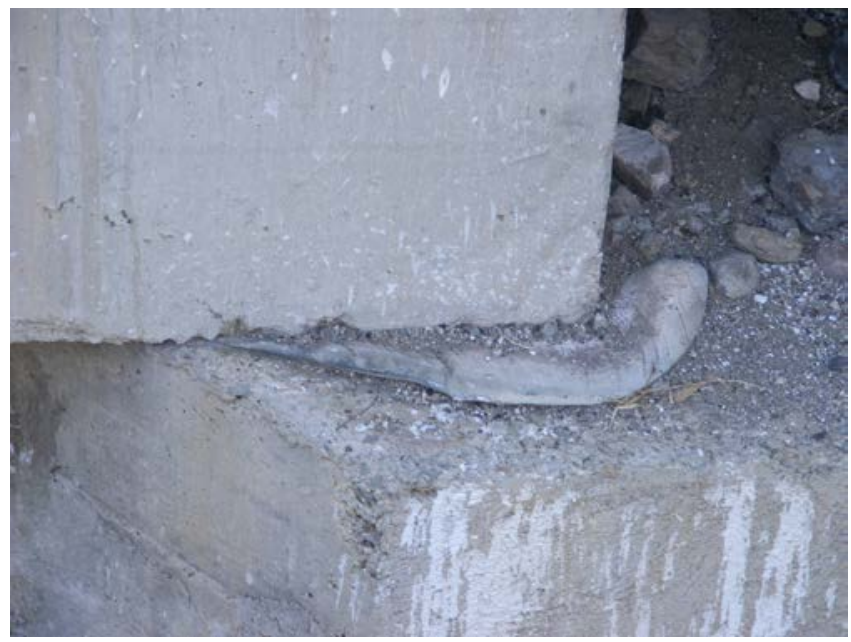

Figura 10. Aparatos de apoyo inadecuados sobre cabezales de pilas.

evaluadas de altamente vulnerables (puentes 2, 5, 6, 11, 14 y 17). El análisis de este parámetro ha sido priorizado por los códigos y manuales vigentes. Luego de los daños ocurridos en los puentes durante el sismo de febrero del 2010,

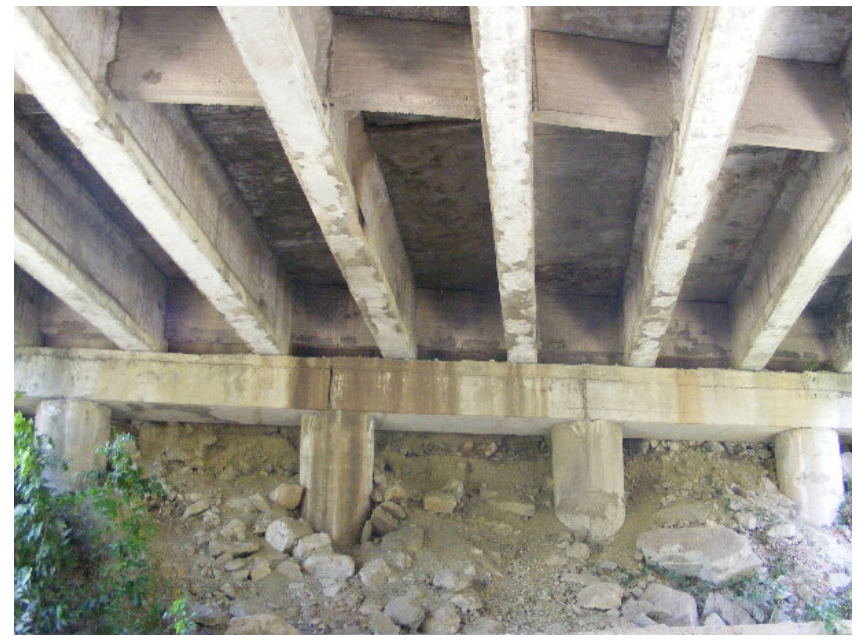

Figura 7. Deslizamiento del material del aproche entre las columnas del estribo.

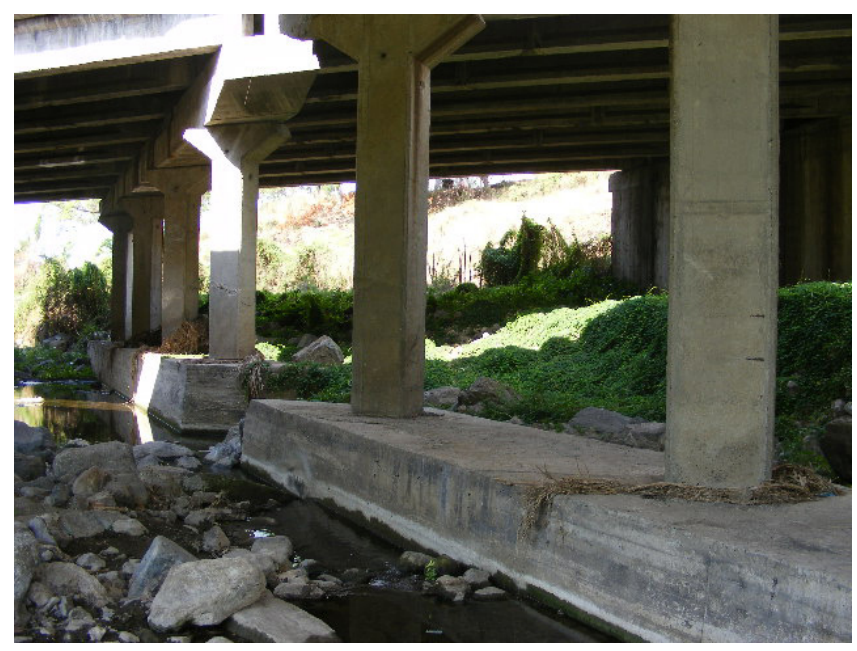

Figura 9. Socavación al pie de pilas que deja al descubierto parte de la cimentación.

(22) instrumentó la consideración de mayores longitudes de asiento de las vigas sobre los cabezales, basados en los criterios de (23).

- Ausencia de trabas sísmicas (topes transversales): la tipología analizada no prevé la construcción de estos elementos, los cuales contribuyen a impedir la caída de los tramos por desplazamientos superiores a los considerados en el diseño

- Ausencia de diafragmas (vigas transversales): solo las poseen los puentes con luces mayores de 24 metros. Los diafragmas incrementan la rigidez transversal de las estructuras e impiden la caída de los tramos.

- Esviaje: las estructuras esviadas con ángulos iguales o superiores a $30^{\circ}$ tienen mayor propensión a ser dañadas por terremotos fuertes (puentes 2, 5, 6, 11, 14 y 17).

- Tipo de suelo: muchos de los puentes estudiados se encuentran en zonas de suelos suaves que amplifican las ondas sísmicas (puentes 1, 2, 3, 4, 5, 6, 8, 11).

- Licuación del terreno: por su ubicación, los puentes situados en suelos suaves y con presencia de agua pueden ser afectados por este fenómeno geológico.

La aplicación de la metodología permite establecer un orden de prioridad para la toma de decisiones en el proceso de rehabilitación del conjunto de estructuras evaluadas. Al mismo 
Tabla 6. Evaluación del estado técnico de los puentes.

\begin{tabular}{|c|c|c|c|c|c|}
\hline Puente & Estado técnico & Puente & Estado técnico & Puente & Estado técnico \\
\hline 01 & 4 (bien) & 07 & 3 (regular) & 13 & 4 (bien) \\
\hline O2 & 4 (bien) & 08 & 3 (regular) & 14 & 4 (bien) \\
\hline 03 & 3 (regular) & 09 & 3 (regular) & 15 & 3 (regular) \\
\hline 04 & 3 (regular) & 10 & 3 (regular) & 16 & 4 (bien) \\
\hline 05 & 3 (regular) & 11 & 3 (regular) & 17 & 3 (regular) \\
\hline 06 & 3 (regular) & 12 & 4 (bien) & 18 & 3 (regular) \\
\hline
\end{tabular}

Tabla 7. Información estructural básica para el análisis preliminar de la vulnerabilidad sísmica estructural.

\begin{tabular}{|c|c|c|c|c|}
\hline \multirow[b]{2}{*}{ Puente } & \multicolumn{4}{|c|}{ Información estructural } \\
\hline & $\begin{array}{l}\text { Esviaje } \\
\text { (o) }\end{array}$ & $\begin{array}{l}\text { Gálibo vertical } \\
\text { (m) }\end{array}$ & Tipo de pilas & Tipo de estribos \\
\hline 01 & $15^{\circ}$ & 21.00 & $\begin{array}{l}\text { Pórticos de } 2 \text { columnas } \\
\text { hormigonadas in situ }\end{array}$ & $\begin{array}{l}\text { Abiertos con } 4 \text { pilotes - columnas } \\
\text { circulares }\end{array}$ \\
\hline 02 & $30^{\circ}$ & 7.60 & $\begin{array}{l}\text { Pórticos de } 4 \text { columnas } \\
\text { prefabricadas }\end{array}$ & $\begin{array}{l}\text { Abiertos. Pórticos de } 4 \text { columnas } \\
\text { prefabricadas }\end{array}$ \\
\hline 03 & $\mathrm{O}^{\circ}$ & 12.60 & $\begin{array}{l}\text { Pórticos de } 4 \text { columnas } \\
\text { prefabricadas }\end{array}$ & $\begin{array}{l}\text { Abiertos. Pórticos de } 4 \text { columnas } \\
\text { prefabricadas }\end{array}$ \\
\hline 04 & $\mathrm{O}^{\circ}$ & 11.00 & $\begin{array}{l}\text { Pórticos de } 2 \text { columnas } \\
\text { hormigonadas in situ }\end{array}$ & $\begin{array}{l}\text { Abiertos. Pórticos de } 4 \text { columnas } \\
\text { prefabricadas }\end{array}$ \\
\hline 05 & $30^{\circ}$ & 8.10 & $\begin{array}{l}\text { Pórticos de } 4 \text { columnas } \\
\text { prefabricadas }\end{array}$ & $\begin{array}{l}\text { Cerrado con aletones a } 90^{\circ} \text {. En la dirección } \\
\text { Palma son abiertos con } 4 \text { pilotes-columnas } \\
\text { circulares. }\end{array}$ \\
\hline 06 & $45^{\circ}$ & $7 \cdot 50$ & $\begin{array}{l}\text { Pórticos de } 4 \text { columnas } \\
\text { prefabricadas }\end{array}$ & $\begin{array}{l}\text { Abiertos. Pórticos de } 4 \text { columnas } \\
\text { prefabricadas }\end{array}$ \\
\hline 07 & $15^{\circ}$ & 13.50 & $\begin{array}{l}\text { Pórticos de } 2 \text { columnas } \\
\text { hormigonadas in situ }\end{array}$ & Abiertos con 4 pilotes-columnas benoto. \\
\hline 08 & $\mathrm{O}^{\circ}$ & 5.40 & $\begin{array}{l}\text { Pórticos de } 4 \text { columnas } \\
\text { prefabricadas }\end{array}$ & Abiertos con 4 pilotes-columnas circulares \\
\hline 09 & $\mathrm{O}^{\circ}$ & 4.00 & $\begin{array}{l}\text { Pórticos de } 4 \text { columnas } \\
\text { prefabricadas }\end{array}$ & $\begin{array}{l}\text { Cerrados con piezas prefabricadas, aletones } \\
\text { a } 90^{\circ} \text {. }\end{array}$ \\
\hline 10 & $15^{\circ}$ & 4.50 & $\begin{array}{l}\text { Pórticos de } 4 \text { columnas } \\
\text { prefabricadas }\end{array}$ & Abiertos con 4 pilotes-columnas circulares \\
\hline 11 & $30^{\circ}$ & 8.50 & $\begin{array}{l}\text { Pórticos de } 4 \text { columnas } \\
\text { prefabricadas }\end{array}$ & $\begin{array}{l}\text { Dirección Este cerrado y fundido in situ. } \\
\text { En la dirección Oeste, abierto con } 4 \text { columnas } \\
\text { prefabricadas. }\end{array}$ \\
\hline 12 & $15^{\circ}$ & $7 \cdot 50$ & $\begin{array}{l}\text { Pórticos de } 4 \text { columnas } \\
\text { prefabricadas }\end{array}$ & Abiertos con 4 pilotes-columnas circulares \\
\hline 13 & $\mathrm{O}^{\circ}$ & 6.30 & $\begin{array}{l}\text { Pórticos de } 4 \text { columnas } \\
\text { prefabricadas }\end{array}$ & $\begin{array}{l}\text { Cerrados con piezas prefabricadas, aletones } \\
\text { a } 90^{\circ} \text {. }\end{array}$ \\
\hline 14 & $30^{\circ}$ & $7 \cdot 50$ & $\begin{array}{l}\text { Pórticos de } 4 \text { columnas } \\
\text { prefabricadas }\end{array}$ & $\begin{array}{l}\text { Abiertos. Pórticos de } 4 \text { columnas } \\
\text { prefabricadas }\end{array}$ \\
\hline 15 & $15^{\circ}$ & 20.00 & $\begin{array}{l}\text { Pórticos de } 2 \text { columnas } \\
\text { hormigonadas in situ }\end{array}$ & $\begin{array}{l}\text { Pórticos de } 2 \text { columnas hormigonadas } \\
\text { in situ }\end{array}$ \\
\hline 16 & $\mathrm{O}^{\circ}$ & 6.60 & $\begin{array}{l}\text { Pórticos de } 4 \text { columnas } \\
\text { prefabricadas }\end{array}$ & $\begin{array}{l}\text { Abiertos. Pórticos de } 4 \text { columnas } \\
\text { prefabricadas }\end{array}$ \\
\hline 17 & $45^{\circ}$ & 7.10 & $\begin{array}{l}\text { Pórticos de } 4 \text { columnas } \\
\text { prefabricadas }\end{array}$ & $\begin{array}{l}\text { Cerrados con piezas prefabricadas, aletones } \\
\text { a } 90^{\circ} \text {. }\end{array}$ \\
\hline 18 & $\mathrm{O}^{\circ}$ & 5.80 & $\begin{array}{l}\text { Pórticos de } 4 \text { columnas } \\
\text { prefabricadas }\end{array}$ & $\begin{array}{l}\text { Abiertos. Pórticos de } 4 \text { columnas } \\
\text { prefabricadas }\end{array}$ \\
\hline
\end{tabular}

tiempo, es posible adoptar medidas rápidas para disminuir el nivel de vulnerabilidad detectado. En este caso, teniendo en cuenta la incidencia de las características el tablero en el resultado obtenido, se recomienda la construcción de una losa de hormigón armado (24) que actúe como diafragma en todo el tablero, respetando las juntas constructivas necesarias. Esta medida contribuiría a:

- Garantizar la distribución de las cargas entre los tramos isostáticos de la estructura. En la dirección longitudinal se le confiere continuidad al tablero, mejorando su comportamiento.
En la dirección vertical se mantiene su comportamiento isostático pues la inercia de la nueva losa es mucho menor que la inercia de las vigas en esa dirección y por tanto no se modifica el comportamiento estructural concebido en el diseño.

- Garantiza la unión entre los tramos y por tanto disminuye el riesgo de pérdida de soporte de estos por la escasa longitud de apoyo. Asimismo incrementa la rigidez en la dirección longitudinal al garantizar el trabajo conjunto de las pilas ante las acciones sísmicas.

- Incrementa el confort de la circulación al disminuir la cantidad de juntas que posee el tablero isostático. 
Tabla 8. Resultados del análisis preliminar de la vulnerabilidad sísmica estructural (IVSE).

Puntuación obtenida en la evaluación de cada grupo de parámetros.

\begin{tabular}{|c|c|c|c|c|c|}
\hline Puente & Puntuación & VSE & Puente & Puntuación & VSE \\
\hline \multirow{4}{*}{01} & VSpE: 0.725 & \multirow{4}{*}{ Alta } & \multirow{4}{*}{10} & VSpE: 0.75 & \multirow{4}{*}{ Moderada } \\
\hline & VSbE: 0.275 & & & VSbE: 0.275 & \\
\hline & VSt: 0.52 & & & VSt: 0.40 & \\
\hline & IvSE: 0.46 & & & IvSE: 0.43 & \\
\hline \multirow{4}{*}{02} & VSpE: 0.775 & \multirow{4}{*}{ Alta } & \multirow{4}{*}{11} & VSpE: 0.675 & \multirow{4}{*}{ Alta } \\
\hline & VSbE: 0.30 & & & VSbE: 0.325 & \\
\hline & VSt: 0.68 & & & VSt: 0.50 & \\
\hline & IvSE: 0.53 & & & IvSE: 0.47 & \\
\hline \multirow{4}{*}{03} & VSpE: 0.55 & \multirow{4}{*}{ Moderada } & \multirow{4}{*}{12} & VSpE: 0.65 & \multirow{4}{*}{ Alta } \\
\hline & VSbE: 0.225 & & & VSbE: 0.275 & \\
\hline & \begin{tabular}{|l|} 
VSt: 0.52 \\
\end{tabular} & & & \begin{tabular}{|l|} 
VSt: 0.62 \\
\end{tabular} & \\
\hline & IvSE: 0.40 & & & IvSE: 0.47 & \\
\hline \multirow{4}{*}{04} & VSpE: 0.55 & \multirow{4}{*}{ Moderada } & \multirow{4}{*}{13} & VSpE: 0.55 & \multirow{4}{*}{ Moderada } \\
\hline & VSbE: 0.325 & & & VSbE: 0.25 & \\
\hline & VSt: 0.52 & & & VSt: 0.55 & \\
\hline & IvSE: 0.44 & & & IvSE: 0.42 & \\
\hline \multirow{4}{*}{05} & VSpE: 0.775 & \multirow{4}{*}{ Alta } & \multirow{4}{*}{14} & VSpE:0.775 & \multirow{4}{*}{ Alta } \\
\hline & VSbE: 0.325 & & & VSbE: 0.30 & \\
\hline & VSt: 0.52 & & & VSt: 0.45 & \\
\hline & IvSE: 0.50 & & & IvSE: 0.46 & \\
\hline \multirow{4}{*}{06} & VSpE: 0.775 & \multirow{4}{*}{ Alta } & \multirow{4}{*}{15} & VSpE: 0.65 & \multirow{4}{*}{ Moderada } \\
\hline & VSbE: 0.30 & & & VSbE: 0.275 & \\
\hline & \begin{tabular}{|l|} 
VSt: 0.50 \\
\end{tabular} & & & \begin{tabular}{|l|} 
VSt: 0.50 \\
\end{tabular} & \\
\hline & IvSE: 0.48 & & & IvSE: 0.44 & \\
\hline \multirow{4}{*}{07} & VSpE: 0.65 & \multirow{4}{*}{ Alta } & \multirow{4}{*}{16} & VSpE: 0.55 & \multirow{4}{*}{ Alta } \\
\hline & VSbE: 0.275 & & & VSbE: 0.225 & \\
\hline & VSt: 0.65 & & & VSt: 0.8 & \\
\hline & IvSE: 0.48 & & & IvSE: 0.48 & \\
\hline & VSpE: 0.55 & & & VSpE: 0.85 & \\
\hline 08 & \begin{tabular}{|l|} 
VSbE: 0.225 \\
\end{tabular} & Moderada & & \begin{tabular}{|l|} 
VSbE: 0.325 \\
\end{tabular} & Alta \\
\hline 08 & VSt: 0.52 & noderada & 17 & VSt: 0.60 & Alta \\
\hline & IvSE: 0.40 & & & IvSE: 0.54 & \\
\hline & VSpE: 0.55 & & & VSpE: 0.625 & \\
\hline 00 & VSbE: 0.25 & Moderada & 18 & VSbE: 0.225 & Moderada \\
\hline 09 & \begin{tabular}{|l|} 
VSt: 0.52 \\
\end{tabular} & nouerada & 10 & VSt: 0.45 & inoderada \\
\hline & IvSE: 0.41 & & & IvSE: 0.40 & \\
\hline
\end{tabular}

No obstante esta medida debe ser analizada en detalle, pues su implementación incrementa en aproximadamente $6 \%$ el peso de la estructura y por tanto puede incidir negativamente en su cortante basal.

\section{CONCLUSIONES}

1. Se obtuvo una metodología, validada por criterio de expertos, que permite considerar en el análisis de la vulnerabilidad sísmica de puentes de hormigón, los parámetros que inciden directamente en su comportamiento y que no necesita para su aplicación de información de proyecto de la estructura analizada.

2. La herramienta elaborada incluye la evaluación de parámetros que no han sido usualmente analizados por metodologías existentes pero que inciden directamente en el comportamiento de las estructuras. Conjuntamente se definen parámetros invalidantes que permiten resaltar aquellos elementos que por sí solos definen un elevado ni- vel de vulnerabilidad sísmica. Estos aspectos le introducen novedad científica al análisis realizado.

3. La metodología presentada ha sido aplicada en la evaluación de 18 puentes prefabricados de hormigón, arrojando que el $56 \%$ de estas estructuras poseen una elevada vulnerabilidad sísmica estructural. Este resultado está determinado por la fecha de diseño y construcción, la existencia de esviajes entre $30^{\circ}$ y $45^{\circ}$, el incumplimiento de la dimensión mínima de la longitud de apoyo establecida por el código de diseño cubano y su ubicación en áreas con suelos suaves que amplifican las acciones sísmicas y son propensos a la licuación.

\section{AGRADECIMIENTOS}

Al Ingeniero Pedro Pablo Torres Rodríguez por su contribución durante la ejecución de su trabajo de diploma. Al Dr. Tomás Jacinto Chuy Rodríguez por sus acertadas sugerencias. A los profesionales que sirvieron de expertos para la validación de la metodología. 


\section{REFERENCIAS}

(1) Kawashima, K. (1990). Present earthquake engineering efforts to mitigate earthquake hazards of road transportation facilities in Japan. San Francisco, U.S.A: ASCE San Francisco 1990 annual Civil Engineering Convention y Exposition.

(2) Kawashima, K., Unjoh, S. (2004). Seismic design of highway bridges. Journal of Japan Association for Earthquake Engineering, 4(3) (Special Issue): 174-183.

(3) Priestley, M., Seible, F., Calvi, G. M. (1996). Seismic Design and retrofit of Bridges, p. 3-29, USA: Editorial John Wiley and Sons.

(4) Keever, M. (2008, 22-24 de septiembre). Caltrans evolving seismic design practice. 24th US-Japan Bridge Engineering Workshop. Recuperado de https://www.pwri.go.jp/eng/ujnr/tc/g/24bws/agenda.htm

(5) Aguiar, R. (2011). El mega sismo de Chile 2010 y lecciones para el Ecuador, p. 130-155, Quito, Ecuador: Sección Nacional del Ecuador del Instituto de Geografía e Historia.

(6) Leyva, K. (2004): Evaluación de la vulnerabilidad sísmica de los puentes de la circunvalación (Tesis de maestría no publicada). Santiago de Cuba: Universidad de Oriente.

(7) Candebat, D., Godínez, G., Caballero, L.F. (2012). Métodos experimentales para la estimación de la vulnerabilidad sísmica de puentes prefabricados de hormigón: «Puente Arroyito». Revista de la Facultad de Ingeniería U.C.V, 27(2): 52-66.

(8) Maldonado, E., Casas, J. R., Canas, J. A. (2001). Modelo de evaluación del índice de vulnerabilidad sísmica de puentes basado en conjuntos difusos. Revista Intern. Ingeniería Estructural, 6: 77-102.

(9) FHWA (2014). LRFD Seismic analysis y design of bridges [Reference manual. Publication No. FHWA-NHI-15-004]. Federal Highway Administration (FHWA).

(10) Federal Emergency Management Agency [FEMA] - National Institute of Building Sciences [NIBS]. (1999).Earthquake loss estimation methodology HAZUS®99, Technical Manual. Washington, D.C.: autor.

(11) Piña, F. (2004). Evaluación de la vulnerabilidad sísmica de puentes carreteros. 12 pp. Revista BIT. Chile.

(12) Jara, J.M., Jara, M., Olmos, B., López, J. (2010, 2 - 4 de junio). Criterio de selección de estructuras tipo de puentes para su evaluación sísmica. En Memorias del VI Congreso internacional sobre patología y recuperación de estructuras (pp. 1-21). Córdoba, Argentina:

(13) Ritta, R. J., Suárez, L.E. y Pando, M.A. (2012). Determinación del período fundamental del suelo usando vibración ambiental y el cociente espectral horizontal/vertical. Mecánica computacional, XXXI, 1399-1419.

(14) American Association of State Highway and Transportation Officials [AASHTO]. (2012). LRFD Bridges. Design specifications (6th edition). Washington, DC, USA: autor.

(15) Linstone, H. A., y Turof, M. (Eds.). (2002). The Delphi method. Techniques and Applications. Boston. Addison-Wesley publishing.

(16) López, E. (2016). El método Delphi en la investigación actual en educación: una revisión teórica y metodológica. Educación XXI, 21 (1): 17-40.

(17) Reguant-Álvarez, M. y Torrado-Fonseca, M. (2016). El método Delphi. REIRE, Revista d’Innovació i Recerca en Educació, 9 (1), 87-102. DOI: 10.1344/reire2016.9.1916

(18) NC. (2003). Norma Cubana. NC 207:2003: Requisitos generales para el diseño y construcción de estructuras de hormigón. Oficina Nacional de Normalización.

(19) ITALCONSULT (1973). Proyecto típico. Tipología cubano italiana. La Habana.

(20) NC. (2004). Norma Cubana. NC 335:2004. Inspección y Conservación de puentes. Códigos de buenas prácticas. Oficina Nacional de Normalización.

(21) NC. (1999). Norma Cubana NC 46:1999: Construcciones Sismorresistentes. Requisitos Básicos para el Diseño y Construcción. Oficina Nacional de Normalización.

(22) Ministerio de Obras Públicas [MOP]. Dirección de Vialidad. Departamento de proyectos de estructuras. (2016). Nuevos criterios sísmicos para el diseño de puentes en Chile [versión G]. Santiago de Chile: autor.

(23) Japan Road Association [JRA]. (2012). Japanese Codes for Design of Highway Bridges. Specifications of Highway Bridges. Part V: Seismic Design. Japón: autor.

(24) Sauter, F. (1993). Desplazamientos: Consideración prioritaria en el diseño sismorresistente de puentes. En memorias del VIII seminario latinoamericano de Ingeniería sismorresistente y Primeras jornadas andinas de Ingeniería estructural. Mérida. Venezuela. 